\title{
Benign Cementoblastoma: Revisiting Rare Tumor of Mandible
}

\author{
Sukhpreet Kaur ${ }^{1}$, Tejinder Kaur ${ }^{2}$, Amit Dhawan ${ }^{3}$, Ramandeep Singh Bhullar ${ }^{4}$, Sarika Kapila ${ }^{5}$
}

\begin{abstract}
Benign cementoblastoma was acknowledged by World Health Organization as an odontogenic tumor originating from cementum. Nowadays, it has been included into tumors of mesenchyme and/or odontogenic ectomesenchyme origin, with or without odontogenic epithelium. This article reports an unusual case of cementoblastoma in a 16-year-old male patient, which involved right mandibular first and second molars causing sharp spontaneous pain radiating to temporal region along with local swelling, which was progressive in nature since 3 months. The lesion showed slow growth and was usually asymptomatic; however, pain and swelling were also been reported in a plethora of cases including the one in this article. Radiographically, the lesion commonly highlights a radiopaque mass often merged with the roots of a tooth, which was enveloped and limited peripherally by a radiolucent halo. The treatment involves surgical excision of the mass along with extraction of involved teeth.

Keywords: Cementoblastoma, Lesion, Odontogenic, Radiolucent halo, Rare tumor.

AMEI's Current Trends in Diagnosis \& Treatment (2019): 10.5005/jp-journals-10055-0079
\end{abstract}

\section{INTRODUCTION}

Cementoblastoma (true cementoma) is a relatively rare, benign, slow developing ectomesenchymal odontogenic neoplasm of the jaw defined by the production of cementum mass continuous with the tooth roots of permanent mandibular posterior teeth. Recognized first by Dewey in 1927, its prevalence is higher in young male adults under 30 years of age, and it constitutes $<1-6.2 \%$ of all odontogenic tumors. ${ }^{1}$ There has been no significant sex predilection. The lesions vary in size between $0.5 \mathrm{~cm}$ and $5.5 \mathrm{~cm}$. Pain and swelling are the most common findings in the patients. Radiographically, a radiopaque mass is usually adhered to the apices of the roots of the teeth. Microscopically, it appears as irregular trabeculae of cementum enclosed by cementoblasts and radiating trabeculae at the periphery with well-defined borders. ${ }^{2}$ Histologically, it is a well-circumscribed tumor with cementum-like tissues surrounded by a fibrous capsule.

The treatment consists of excision of the tumor along with extraction of the affected tooth/teeth. On the contrary, conservative treatment of cementoblastoma, for instance, endodontic therapy and apicoectomy of the involved teeth have also been stated. Reported recurrence rate of cementoblastoma is $21.7 \% .^{3}$

We report a case of benign cementoblastoma in a 16-year-old male patient who presented with a densely mineralized mass seen at apices of mandibular right first and second molars.

\section{Case Description}

A 16-year-old male patient came to the department of oral and maxillofacial surgery with pain and progressive swelling in the right lower posterior region since 3-4 months. No past medical or maxillofacial trauma history was reported. On extraoral examination, a diffuse swelling involving right lower one third of face was observed, which was tender and bony hard on palpation. Intraorally, the lesion was extending from right mandibular first molar to mesial aspect of mandibular second molar, showing mild obliteration of the buccal vestibule. Electric pulp testing revealed vitality of all the teeth associated with the lesion. No mobility or neurosensory deficit was elicited clinically.
${ }^{1-5}$ Department of Oral Surgery, Sri Guru Ram Das Institute of Dental Sciences and Research, Amritsar, Punjab, India

Corresponding Author: Sukhpreet Kaur, Department of Oral Surgery, Sri Guru Ram Das Institute of Dental Sciences and Research, Amritsar, Punjab, India, Phone: +918360199192, e-mail: sukhivirk82@gmail. com

How to cite this article: Kaur S, Kaur T, Dhawan A, et al. Benign Cementoblastoma: Revisiting Rare Tumor of Mandible. AMEl's Curr Trends Diagn Treat 2019;3(2):86-89.

Source of support: Nil

Conflict of interest: None

Panoramic radiographs and computed tomographic (CT) scan were taken for radiological examination. Orthopantomogram showed a mixed radiolucent-radiopaque mass with well-defined radiolucent rim in relation to the right permanent mandibular first and second molars, which further led to resorption of distal root of right mandibular first molar and mesial root of mandibular second molar (Fig. 1).

The CT scan of mandible of the patient revealed well-defined lobulated expansile predominantly radiodense lesion in relation to the apices of right mandibular first molar tooth (Fig. 2). With these clinical findings, history, and radiological findings, provisional diagnosis of benign tumor of the jaw, with cementoblastoma being the first choice in differential diagnosis, was made because of the age, location, and vitality of the teeth. The differential diagnosis included juvenile ossifying fibroma, hypercementosis, condensing osteitis, fibro-osseous lesions, ameloblastoma, and bone neoplasm (mainly osteoblastoma).

Using intraoral approach, under general anesthesia, the calcified mass was enucleated along with the extraction of the involved teeth $(46,47)$ (Figs 3 to 5 ) followed by copious irrigation. Soft tissue healing occurred by 3 weeks, and no recurrence was seen clinically or radiographically 6 months postoperatively.

Macroscopically, it presented as two hard ovoid masses with calcified material measuring $0.5 \mathrm{~cm} \times 0.5 \mathrm{~cm}$ in diameter. Microscopically, it showed calcified cementum-like tissue which was 


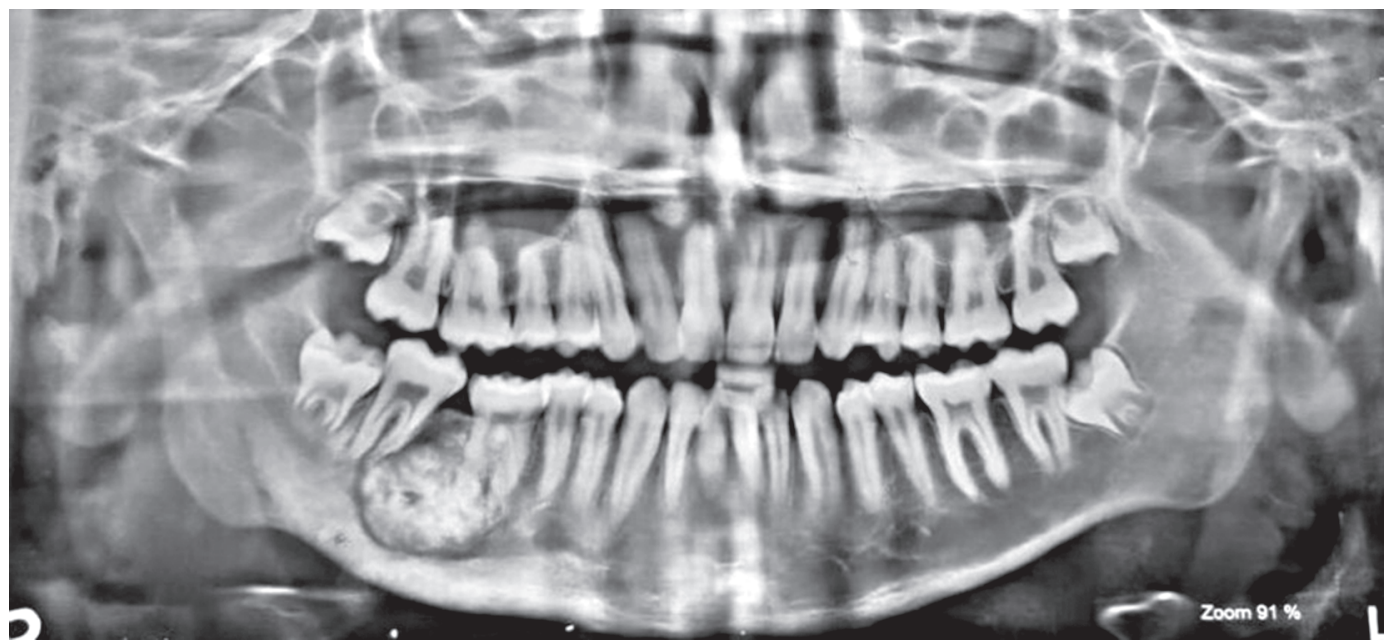

Fig. 1: Panoramic radiograph showing a mixed radiopaque-radiolucent mass with well-defined borders surrounded by a radiolucent rim

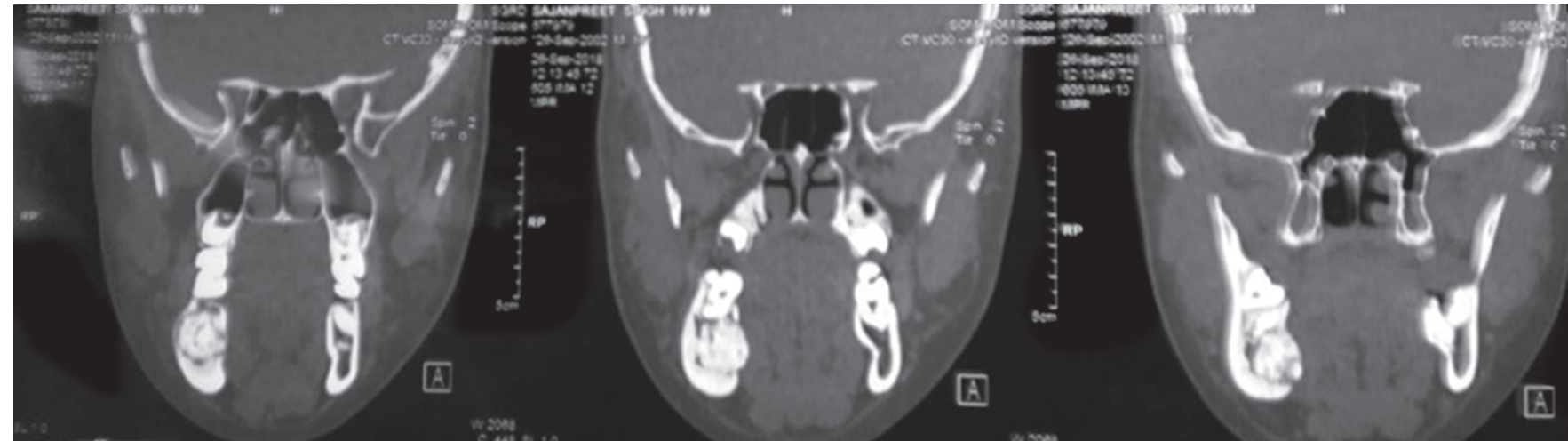

Fig. 2: Coronal section revealed well-defined lobulated radiodense lesion in relation to the root of the lower right first molar

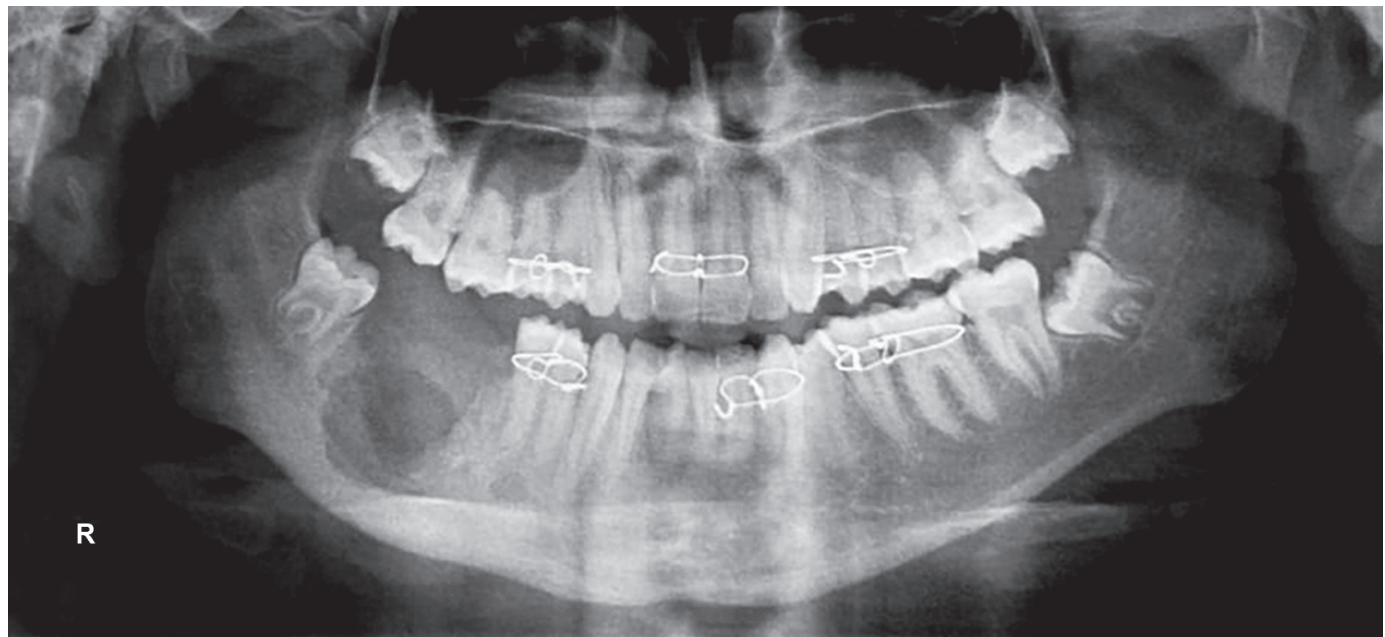

Fig. 3: Panoramic radiograph showing enucleation of calcified mass with extraction of the tooth involved $(46,47)(1$ st day postoperative)

basophilic, and in-between fibrovascular stroma with blood vessels were identified. At few places, lining by cementoblasts were seen which were also found scantly in the stroma (Fig. 6).

\section{Discussion}

Cementoblastoma is an odontogenic tumor obtained from ectomesenchymal cells of the periodontium incorporating cementoblasts. Although slow growing, it had immense growth potential. The male to female ratio has been reported to be 2.1:1 with a mean prevalence of 20.7 years age group. It shows more prevalence in mandible involving erupted permanent first mandibular molar. The most significant clinical symptom is painful swelling which is found at the buccal and lingual/ palatal aspects of the alveolar ridges; sometimes it could be 


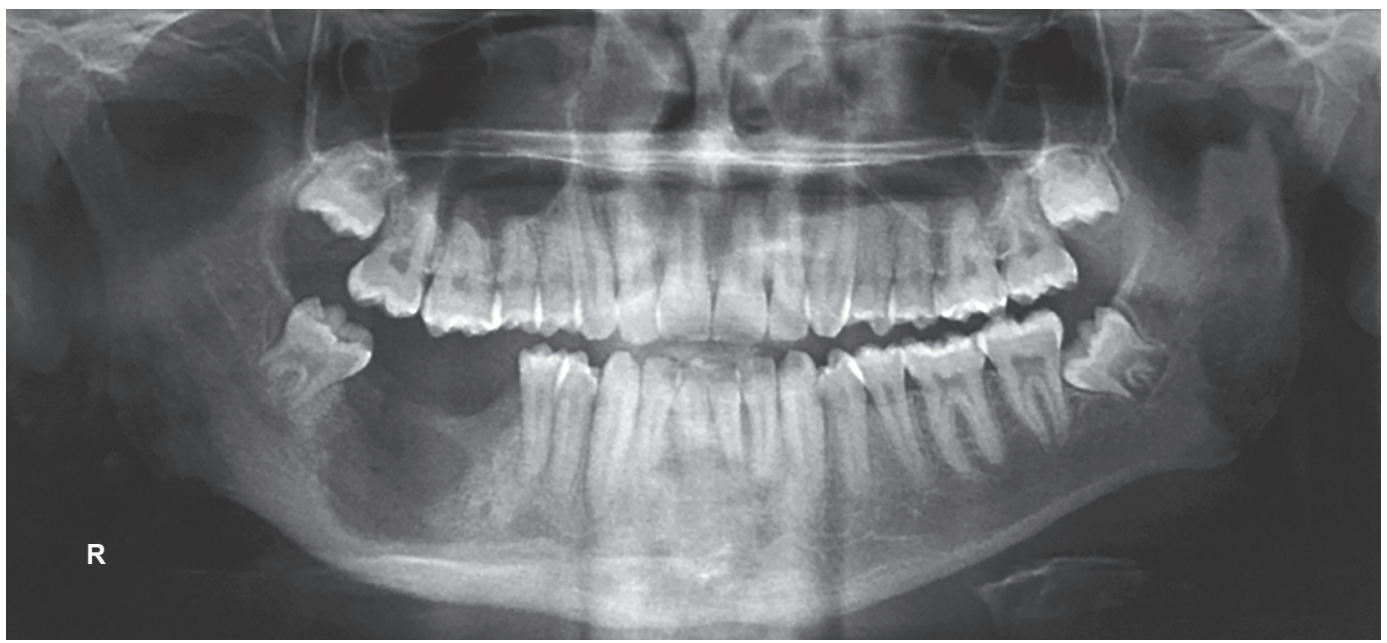

Fig. 4: 1st postoperative month panoramic radiograph

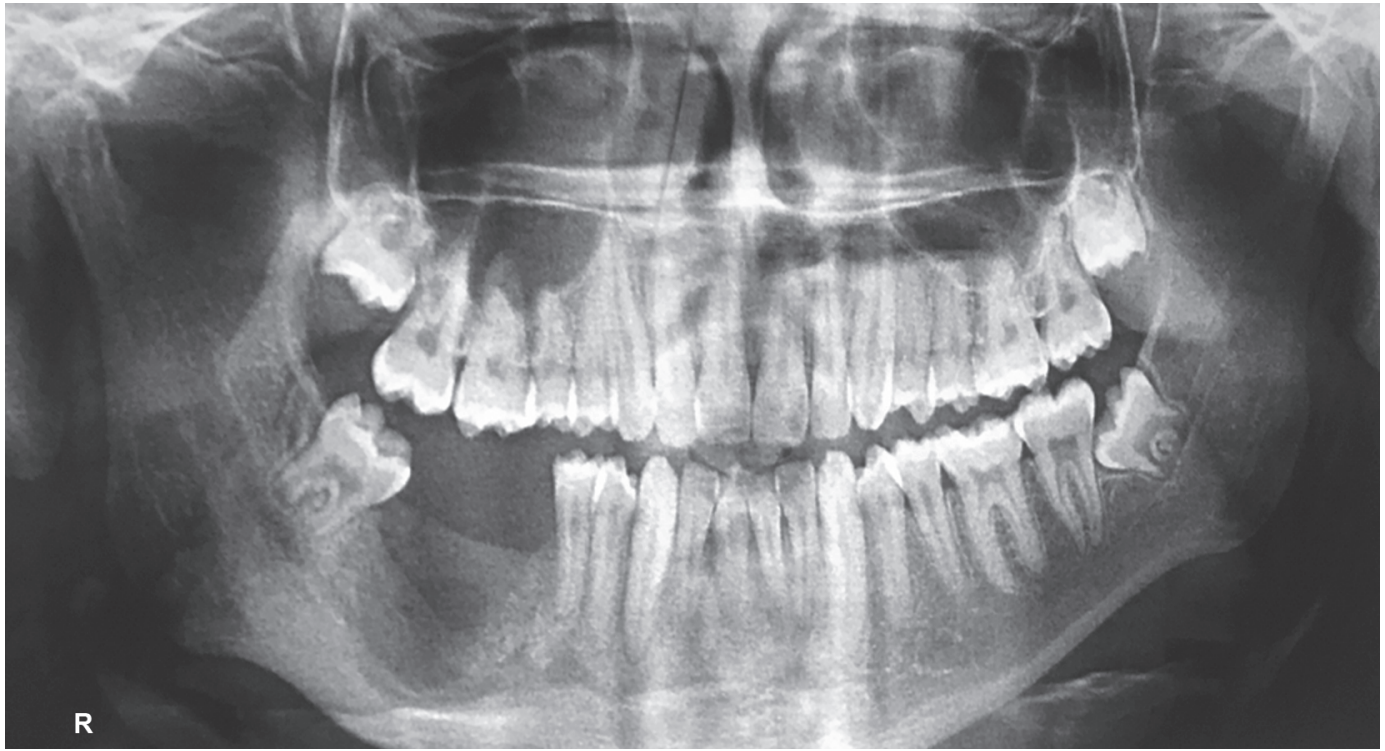

Fig. 5: 6th postoperative month panoramic radiograph

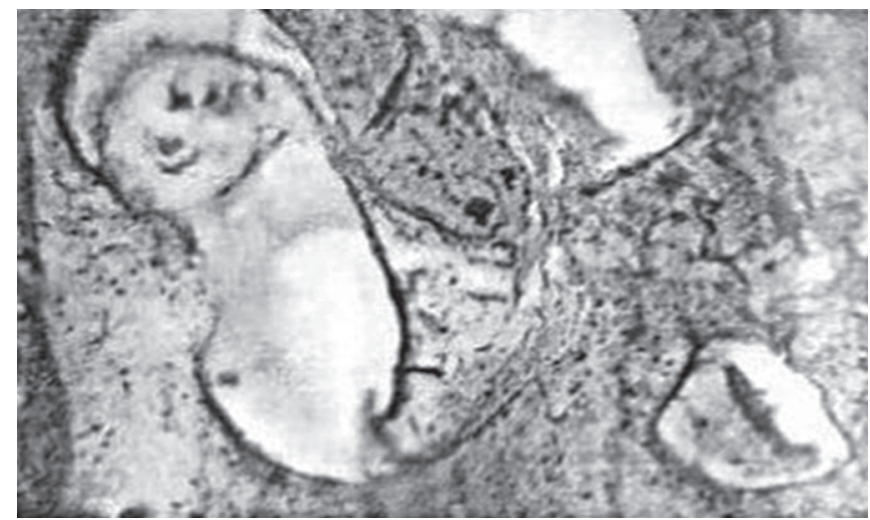

Fig. 6: Microphotograph revealed sheets of cementum-like tissues consisting of cementoblasts

symptomless. ${ }^{4}$ The associated tooth or teeth usually shows normal pulp vitality score. It frequently shows cortical expansion and asymmetry of face. On the contrary, rare reported evidence are available of lower lip paresthesia or pathologic fracture of the mandible associated with tumor.

Although it is a benign tumor, aggressive behavior has been reported. Some cases reported in the literature depict that it shows localized bony destruction which involves expansion of bone, cortical plates erosion, displacement of adjoining teeth, and invasion of the pulp and root canals, and it may also involve adjacent teeth. ${ }^{1}$ Brannon et al. found that the incidence of aggressive nature was more in recurrent tumors than in nonrecurring tumors. The authors reported recurrence in $13(37.1 \%)^{3}$ of 44 cases.

Radiographically, benign cementoblastoma represents as a well-defined radiopacity with a radiolucent zone. ${ }^{1}$ The lesion in early stage appears more radiolucent, and it is difficult to differentiate them from the periapical inflammatory lesions and focal osteomyelitis.

In mature and the late stages, it might be difficult to distinguish this lesion from hypercementosis, cement-ossifying fibroma, osteoma, benign osteoblastoma, odontomas, calcifying epithelial odontogenic tumors, and so on. ${ }^{4}$ The average growth of this 
tumor has been found as $0.5 \mathrm{~cm} /$ year. ${ }^{3}$ Histologically, this tumor shows sheets of cementum-like tissues, in which a large number of reversal lines with active cementoblasts and fibrous connective tissue capsule may be found at periphery. ${ }^{5}$

It resembles osteoblastomas that mostly involve males in second decade of their life. The clinical features of osteoblastoma also present as localized pain and swelling but radiographically osteoblastoma are circumscribed lytic lesions without the typical peripheral radiolucent rim. The classic radiating trabeculae at the periphery and the well-defined borders adjoining normalappearing underlying bone on microscopy are not found in osteoblastoma.

\section{CONCLUSION}

To reiterate, cementoblastoma is a rare odontogenic tumor. Thus, it needs to be considered in the differential diagnosis of periapical radiopacities. The foremost diagnosis is established by its adhesion with the apices of tooth root and radiographic halo surrounding the radiopaque mass. The treatment remains the complete excision of lesion along with the extraction of the involved teeth. The complete removal of the tumor and careful follow-up immensely reduces the recurrence chances.

\section{References}

1. Sharma N. Benign cementoblastoma: a rare case report with review of literature. Contemp Clin Dent 2014;5(1):92. DOI: 10.4103/0976237X.128679.

2. Teixeira LR, dos Santos JL, Almeida LY, et al. Residual cementoblastoma: an unusual presentation of a rare odontogenic tumor. J Oral Maxillofac Surg, Med Path 2018;30(2):187-190. DOI: 10.1016/j. ajoms.2017.11.001.

3. Brannon RB, Fowler CB, Carpenter WM, et al. Cementoblastoma: an innocuous neoplasm? A clinicopathologic study of 44 cases and review of the literature with special emphasis on recurrence. Oral Surg Oral Med Oral Pathol Oral Radiol Endod 2002;93(3):311-320. DOI: 10.1067/moe.2002.121993.

4. Subramani V, Narasimhan M, Ramalingam S, et al. Revisiting cementoblastoma with a rare case presentation. Case Rep Pathol 2017;2017:8248691. DOI: 10.1155/2017/8248691.

5. Napier Souza L, Monteiro Lima Júnior S, Garcia Santos Pimenta FJ, et al. Atypical hypercementosis versus cementoblastoma. Dentomaxillofac Radiol 2004;33(4):267-270. DOI: 10.1259/dmfr/30077628. 